\title{
Cured bronchi! Extending the use of nebulised hypertonic saline outside of cystic fibrosis?
}

\author{
Clémence Martin ${ }^{1,2}$, Lucile Regard ${ }^{1,2}$ and Pierre-Régis Burgel (10 ${ }^{1,2}$ \\ Affiliations: ${ }^{1}$ Université Paris Descartes, Sorbonne Paris Cité, Paris, France. ${ }^{2}$ Dept of Respiratory Medicine \\ and National Reference Center for Cystic Fibrosis Cochin Hospital, Assistance Publique, Hôpitaux de Paris, \\ Paris, France.
}

Correspondence: Pierre-Régis Burgel, Dept of Respiratory Medicine, Cochin Hospital, 27 rue du Faubourg Saint Jacques, 75014 Paris, France. E-mail: pierre-regis.burgeldaphp.fr

@ERSpublications

Hypertonic saline may be effective not only in cystic fibrosis but also in other chronic airway diseases http://ow.ly/dioH30jGXoa

Cite this article as: Martin C, Regard L, Burgel P-R. Cured bronchi! Extending the use of nebulised hypertonic saline outside of cystic fibrosis? Eur Respir J 2018; 51: 1800755 [https://doi.org/10.1183/ 13993003.00755-2018].

\begin{abstract}
Plugging of the airways by excessive mucus is an important contributor to morbidity and mortality in chronic airway diseases (e.g. cystic fibrosis (CF), chronic obstructive pulmonary disease (COPD), bronchiectasis and primary ciliary dyskinesia (PCD)) [1]. Such findings are the basis for the major interest in drugs that reduce mucus content in airways by targeting mucus production [2] or by increasing clearance of mucus [3]. A single aerosol of hypertonic saline (HS) was shown to enhance clearance of airway secretions in humans $>40$ years ago [4], and a randomised control trial reported $>10$ years ago found that long-term nebulisation of HS resulted in decreased rates of pulmonary exacerbations in patients with CF [5]. However, the mechanisms underlying enhanced clearance of airway mucus and clinical benefits have not been fully elucidated [6]. In vitro studies supported the hypothesis that HS increased mucociliary clearance, as the administration of HS increased the volume of airway surface liquid (ASL) in cultured airway epithelial cells, an effect that was larger and more prolonged in cultures from patients with CF than in cultures of controls [7]. Based on these experimental and clinical results, HS is now used in many countries for the treatment of patients with CF [8]. However, there is only limited evidence that HS is effective in other chronic airway diseases. For example, the small number of studies comparing the effects of hypertonic versus isotonic saline in (non-CF) bronchiectasis yielded conflicting results $[9,10]$, and a recent 3-month crossover trial in patients with PCD found no effect on quality of life and lung function [11]. At first glance, it may be concluded that beneficial effects of HS in airway epithelium are limited to CF airways, with only limited effects in non-CF airways, due to altered ion transport in CF epithelium. However, it has been hypothesised that increase in the magnitude of effects of HS in CF versus normal epithelial cells is not related to cystic fibrosis transmembrane conductance regulator dysfunction itself, but to increased water retention [12] by the hyperconcentrated mucus that is found in CF airways [13, 14].

In the current issue of the European Respiratory Journal, GoRALSKI et al. [15] present the results of an original study in which the authors developed an in vitro aerosolisation system in primary cultures of airway epithelial cells grown at the air-liquid interface. Their primary goal was to mimic in vivo aerosol delivery rates of HS to the airway epithelium with the aims of 1) studying the kinetics of HS on airway surface hydration, and 2) proposing strategies to improve efficacy of HS in the treatment of chronic airway
\end{abstract}


diseases. The authors first studied non-CF airway epithelial cells with normal (2\%) mucus content (obtained by daily washing of secreted airway mucus at the apical surface). In this model, they showed that HS nebulisation resulted in rapid increase in ASL height that was paralleled by a decrease in epithelial cell height, suggesting an osmotic transfer of water from epithelial cells to the ASL, at least in part via aquaporin-mediated increase in cellular water permeability. The effect on ASL was only transient, as ASL reabsorption started immediately at the end of the aerosol, and ASL height returned to baseline within $1 \mathrm{~h}$; epithelial cell height did not return to baseline within $4 \mathrm{~h}$ following $\mathrm{HS}$ administration and the effect of a second nebulisation of HS on ASL was blunted, presumably due to the absence of cell volume regulation mechanisms. Interestingly, a nebulisation of hypotonic saline after a first HS nebulisation restored the ASL response to a second HS nebulisation. The effect of various HS nebulisation protocols (mimicking the rates of nebulisation obtained with a jet nebuliser or a vibrating mesh nebuliser) in airway epithelial cells suggested that the currently used nebulisation protocols produced appropriate increase in ASL, whereas slower rates of nebulisation were less effective.

Next, Goralski et al. [15] studied the effects of HS in non-CF airway epithelial cells with high (12\%) ASL mucus content (generated by leaving the apical surface of the epithelial cells unwashed for 2 weeks). The authors showed that the change in ASL height following nebulisation of HS was markedly increased in hyperconcentrated (12\%) mucus cultures compared to normal (2\%) mucus cultures. ASL reabsorption, which also started immediately after the end of nebulisation, was slower in hyperconcentrated mucus cultures and total duration of ASL volume expansion was approximately double that of normal mucus cultures. In marked contrast with normal mucus cultures, a second nebulisation of HS produced an ASL response similar to the first administration. These effects in non-CF cells with hyperconcentrated mucus were comparable to those previously reported in CF cells. Based on these findings, the authors suggested that repetitive HS dosing, which is currently used in CF patients [5], may be an efficient strategy in patients with highly concentrated mucus contents (presumably those patients with severe diseases, including CF), but that other strategies (e.g. delivering hypotonic saline in between HS nebulisations) may be required to optimise the effectiveness of HS in patients with lower mucus contents (and milder diseases).

The studies by GoRALSKI et al. [15] are most welcome in that they provide interesting insights into the mechanistic effects underlying the effects of HS in non-CF airway epithelium and suggest the possibility of extending the use of HS from CF to other chronic airway diseases, including bronchiectasis and COPD. However, several limits may exist to this proposal. First, HS nebulisation has been extensively studied in COPD patients, in whom it can be used to perform sputum induction [16]. Several studies have documented the risk of adverse airway reaction, characterised by increased dyspnoea, decreased oxygen saturation and/or clinically significant decrease in forced expiratory volume in $1 \mathrm{~s}[17,18]$. Although these effects were generally regarded as mild in the context of experimental sputum induction in a medical setting, they occurred frequently, which raises concerns on the possibility of using long-term HS in patients with COPD at home. Secondly, although the suggestion of using both hypertonic and hypotonic saline in sequential ways for restoring the response of airway epithelium to HS in patients with milder diseases is appealing, the safety of this strategy will have to be tested as both hypertonic and hypotonic saline were reported to induce bronchoconstriction in patients with asthma [19]. Finally, the findings by GoRALSKI et al. [15] that HS nebulisation induces the release of interleukin (IL)-8, a potent neutrophil chemoattractant, from cultured epithelial cells could be a matter of concern. Comparable findings were also reported in animal studies in mice [20] and HS nebulisation has been suggested to promote the release of preformed inflammatory mediators from airway epithelial and/or mast cells in human airways [17, 21]. However, IL-8 levels in sputum did not differ after isotonic saline versus HS inhalation in patients with COPD [22], and HS has also been reported to promote proteolytic degradation of IL-8 in CF airways, resulting in decreased IL-8 content [23]. Thus, we suggest that the clinical meaning of IL-8 increase by HS in experimental models remains currently unknown.

In the end, the elegant in vitro studies by Goralski et al. [15] suggest that 1) the beneficial effect of HS may not be limited to CF and could be extended to other chronic airway diseases in which mucus hypersecretion occurs, and 2) administration regimens used in CF may not be strictly transposable to other chronic airway diseases, especially in milder forms of disease with less concentrated airway mucus. To date, clinical trials performed in diseases other than CF have lacked sufficient number of patients and/or evaluated only relatively short-term periods of treatment with outcomes such as lung function and/or quality of life. Appropriate long-term clinical trials will be necessary to confirm the hypothesis that "curing" (i.e. preserving by adding salt) the bronchi will result in increased mucociliary clearance and meaningful clinical benefits (e.g. improvement in pulmonary exacerbations) in patients with non-CF chronic airway diseases.

Conflict of interest: C. Martin reports personal fees from Chiesi and Zambon, outside the submitted work. P-R. Burgel reports personal fees from Astra-Zeneca, Boehringer Ingelheim, Chiesi, GSK, Novartis, Teva and Vertex, outside the submitted work. 


\section{References}

$1 \quad$ Fahy JV, Dickey BF. Airway mucus function and dysfunction. N Engl J Med 2010; 363: $2233-2247$.

2 Martin C, Frija-Masson J, Burgel PR. Targeting mucus hypersecretion: new therapeutic opportunities for COPD? Drugs 2014; 74: 1073-1089.

3 Tarrant BJ, Le Maitre C, Romero L, et al. Mucoactive agents for chronic, non-cystic fibrosis lung disease: a systematic review and meta-analysis. Respirology 2017; 22: 1084-1092.

4 Pavia D, Thomson ML, Clarke SW. Enhanced clearance of secretions from the human lung after the administration of hypertonic saline aerosol. Am Rev Respir Dis 1978; 117: 199-203.

5 Elkins MR, Robinson M, Rose BR, et al. A controlled trial of long-term inhaled hypertonic saline in patients with cystic fibrosis. N Engl J Med 2006; 354: 229-240.

6 Ratjen F. Restoring airway surface liquid in cystic fibrosis. N Engl J Med 2006; 354: 291-293.

7 Donaldson SH, Bennett WD, Zeman KL, et al. Mucus clearance and lung function in cystic fibrosis with hypertonic saline. N Engl J Med 2006; 354: 241-250.

8 Zolin A, Orenti A, Naehrlich L, et al. ECFS Patient Registry Annual Data Report, 2015 data. Karup, European Cystic Fibrosis Society, 2017. Available from: www.ecfs.eu/projects/ecfs-patient-registry/annual-reports

9 Polverino E, Goeminne PC, McDonnell MJ, et al. European Respiratory Society guidelines for the management of adult bronchiectasis. Eur Respir J 2017; 50: 1700629.

10 Hart A, Sugumar K, Milan SJ, et al. Inhaled hyperosmolar agents for bronchiectasis. Cochrane Database Syst Rev 2014; 5: CD002996.

11 Paff T, Daniels JM, Weersink EJ, et al. A randomised controlled trial on the effect of inhaled hypertonic saline on quality of life in primary ciliary dyskinesia. Eur Respir J 2017; 49: 1601770

12 Bennett WD, Henderson AG, Donaldson SH. Hydrator therapies for chronic bronchitis. Lessons from cystic fibrosis. Ann Am Thorac Soc 2016; 13: Suppl. 2, S186-S190.

13 Burgel PR, Montani D, Danel C, et al. A morphometric study of mucins and small airway plugging in cystic fibrosis. Thorax 2007; 62: 153-161.

14 Henderson AG, Ehre C, Button B, et al. Cystic fibrosis airway secretions exhibit mucin hyperconcentration and increased osmotic pressure. J Clin Invest 2014; 124: 3047-3060.

15 Goralski JL, Wu D, Thelin WR, et al. The in vitro effect of nebulised hypertonic saline on human bronchial epithelium. Eur Respir J 2018; 51: 1702652.

16 Rytilä PH, Lindqvist AE, Laitinen LA. Safety of sputum induction in chronic obstructive pulmonary disease. Eur Respir J 2000; 15: 1116-1119.

17 Taube $\mathrm{C}, \mathrm{Holz} \mathrm{O}, \mathrm{Mücke} \mathrm{M}$, et al. Airway response to inhaled hypertonic saline in patients with moderate to severe chronic obstructive pulmonary disease. Am J Respir Crit Care Med 2001; 164: 1810-1815.

18 Makris D, Tzanakis N, Moschandreas J, et al. Dyspnea assessment and adverse events during sputum induction in COPD. BMC Pulm Med 2006; 6: 17

19 Schoeffel RE, Anderson SD, Altounyan RE. Bronchial hyperreactivity in response to inhalation of ultrasonically nebulised solutions of distilled water and saline. Br Med J 1981; 283: 1285-1287.

20 Graeber SY, Zhou-Suckow Z, Schatterny J, et al. Hypertonic saline is effective in the prevention and treatment of mucus obstruction, but not airway inflammation, in mice with chronic obstructive lung disease. Am J Respir Cell Mol Biol 2013; 49: 410-417.

21 Carpagnano GE, Foschino Barbaro MP, Cagnazzo M, et al. Use of exhaled breath condensate in the study of airway inflammation after hypertonic saline solution challenge. Chest 2005; 128: 3159-3166.

22 Cianchetti S, Bacci E, Bartoli ML, et al. Can hypertonic saline inhalation influence preformed chemokine and mediator release in induced sputum of chronic obstructive pulmonary disease patients? Comparison with isotonic saline. Clin Exp Allergy 2007; 37: 1819-1826.

23 Reeves EP, Williamson M, O’Neill SJ, et al. Nebulized hypertonic saline decreases IL-8 in sputum of patients with cystic fibrosis. Am J Respir Crit Care Med 2011; 183: 1517-1523. 